\title{
An innovative cold storage system for donor heart transportation- lessons learned from the first experience in Switzerland
}

\author{
Martin O. Schmiady ${ }^{1 \#}$, Tim Graf ${ }^{1 \#}$, Ahmed Ouda ${ }^{1}$, Raed Aser ${ }^{1}$, Andreas J. Flammer ${ }^{2} \wedge$, Paul R. Vogt $^{1}$, \\ Markus J. Wilhelm ${ }^{1}$ \\ ${ }^{1}$ Clinic for Cardiac Surgery, University Heart Centre, Zurich, Switzerland; ${ }^{2}$ Clinic for Cardiology, University Heart Centre, Zurich, Switzerland \\ Contributions: (I) Conception and design: MO Schmiady, T Graf, MJ Wilhelm; (II) Administrative support: MO Schmiady, T Graf, MJ Wilhelm; \\ (III) Provision of study materials or patients: MO Schmiady, T Graf, MJ Wilhelm; (IV) Collection and assembly of data: MO Schmiady, T Graf, MJ \\ Wilhelm; (V) Data analysis and interpretation: MO Schmiady, T Graf, MJ Wilhelm; (VI) Manuscript writing: All authors; (VII) Final approval of \\ manuscript: All authors. \\ \#These authors contributed equally to this work. \\ Correspondence to: Martin O. Schmiady, MD. Clinic for Cardiac Surgery, University Heart Centre Zurich, University Hospital Zurich, Rämistrasse \\ 100, CH-8091 Zurich, Switzerland. Email: martinoliver.schmiady@usz.ch.
}

Background: The current standard for donor heart preservation consists of cold organ storage in three sequential plastic bags. This technique can cause freezing injuries of the donor heart as the temperature inside the transport box is not monitored routinely. The SherpaPak ${ }^{\mathrm{TM}}$ Cardiac Transport System (CTS) (Paragonix Technologies, Cambridge, MA, USA) aims to resolve this problem by maintaining a controlled preservation temperature between 4 and $8^{\circ} \mathrm{C}$. This study reports the first single-centre experience in Switzerland with this innovative single-use disposable device.

Methods: Between May and December 2020, four heart procurements using SherpaPak ${ }^{\mathrm{TM}}$ CTS were performed at our heart centre. Donor heart preservation fluid and ambient temperature were monitored using the InTempConnect ${ }^{\circledR}$ application (Onset Computer Corporation, Bourne, MA, USA). All patient data were collected retrospectively from the local hospital patient data capture system.

Results: Four recipients of a donor heart preserved with SherpaPak ${ }^{\mathrm{TM}}$ CTS were included in this study (3 male, 1 female). Mean transport distance was $86 \mathrm{~km}$ (range, $45-276 \mathrm{~km}$ ). Mean storage time in the cooler was $73.5 \pm 19.33$ minutes. Mean cold ischemic time was $199.25 \pm 11.67$ minutes. The device kept the average organ temperature between 5.2 and $8.8{ }^{\circ} \mathrm{C}$ and hereby reached the recommended temperature range of $5-10{ }^{\circ} \mathrm{C}$. Modifications of the procurement and storage process provided an optimization of the temperature course in the transportation box. There were no incidents during the transport. Organs transported with this novel storage system showed normal function after transplantation.

Conclusions: The SherpaPak ${ }^{\mathrm{TM}}$ CTS provides constant organ temperatures during transportation, prevents freezing injury and ensures mechanical protection of the graft.

Keywords: Heart transplantation; organ procurement; cold storage; hypothermic injury; graft transport

Submitted Jul 16, 2021. Accepted for publication Oct 22, 2021.

doi: $10.21037 /$ jtd-21-1175

View this article at: https://dx.doi.org/10.21037/jtd-21-1175

^ ORCID: Martin O. Schmiady, 0000-0003-0210-7904; Andreas J. Flammer, 0000-0002-1373-0630.

(C) Journal of Thoracic Disease. All rights reserved. 


\section{Introduction}

Despite technical advances and low complication rates in the latest generation of left ventricular assist devices (LVADs), heart transplantation remains the gold standard for treatment of patients with end-stage heart failure refractory to optimal medical treatment. Although variations exist between transplant centres, procurement and transport techniques are similar and have not changed appreciably since the early era of heart transplantation in the late 1960s. The gold standard for organ transportation after harvesting is cold storage of the heart using a three-bag-technique with the inner bag filled with preservation solution and placement of the package in a box filled with crushed ice (1). Temperature monitoring of the transport bag or the tissue is not performed routinely and, therefore, information on the exact temperature of the donor heart is missing. A constant organ temperature during transport is of utmost importance as a temperature drop below $2{ }^{\circ} \mathrm{C}$ considerably increases the risk of cold injury and frostbite, which can adversely influence organ function (2). The three-bag-technique, recommended by the current International Society of Heart and Lung Transplantation (ISHLT) guidelines (1), can lead to tissue temperatures below $0{ }^{\circ} \mathrm{C}$ and therefore may bear a risk for organ injury (3).

The SherpaPak ${ }^{\mathrm{TM}}$ Cardiac Transport System (CTS) (Paragonix Technologies, Cambridge, MA, USA) aims to resolve this problem by maintaining a controlled preservation temperature between 4 and $8{ }^{\circ} \mathrm{C}$. In May 2020, we introduced this novel transport system into our procurement procedure. The aim of this study is to report our initial experience, highlight pitfalls, and give advice for application.

We present the following article in accordance with the STROBE reporting checklist (available at https://dx.doi. org/10.21037/jtd-21-1175).

\section{Methods}

From May to December 2020, four heart procurements using SherpaPak ${ }^{\text {TM }}$ CTS were performed in four consecutive heart transplantations, after the SherpaPak ${ }^{\mathrm{TM}}$ CTS became available at our centre. Informed consent was obtained from all patients. All procedures were in accordance with the Declaration of Helsinki as revised in 2013. An authorization from the local ethics committee (Kantonale Ethikkommission Zurich, KEK) was not required for this study since it does not fall within the scope of the Human Research Act. A written statement of the KEK is available. The objective of the study was to evaluate if the SherpaPak ${ }^{\mathrm{TM}}$ CTS provides proper cooling of the donor heart during transportation. As outcome measure, the temperature course of the preservation fluid inside the inner canister was monitored continuously during transfer from the donor to the recipient hospital. Also, cardiac performance on echocardiography, biopsy results and survival served as outcome parameters.

All organs were retrieved from donors with confirmed brain death during a multi organ procurement and stored in the disposable SherpaPak ${ }^{\mathrm{TM}}$ CTS according to the manufacturer's recommendations (Figure 1A). Donor hearts were procured with antegrade perfusion of $250 \mathrm{~mL} / \mathrm{m}^{2}$ cold Kirklin cardioplegia $\left(30 \mathrm{mmol} / \mathrm{L} \mathrm{K}^{+}\right)$. The same solution was used as transport medium in the inner sterile organ canister of the SherpaPak ${ }^{\mathrm{TM}}$ CTS (Figure 1B). The aortic arch was fixed to the $\varnothing 18 \mathrm{~mm}$ heart connector (also available in the sizes $\varnothing 10,25.4,32 \mathrm{~mm}$ ) with a $5 \mathrm{~mm}$ mersilene tape and anchored to the lid of the transport system, allowing for free floating of the heart in the cardioplegic solution (Figure 1C,1D). The organ canister was then connected with the outer canister and safely stored in the shipper. After initiating temperature data logging through Sherpa Trak ${ }^{\mathrm{TM}}$, donor heart preservation fluid as well as ambient temperature were monitored using the InTempConnect ${ }^{\circledR}$ application (Onset Computer Corporation, Bourne, MA, USA) for mobile phones (available for iOS and Android). After arrival in the transplant centre, the heart was perfused with $250 \mathrm{~mL} / \mathrm{m}^{2}$ cold cardioplegic solution, before it was implanted. Orthotopic heart transplantation was performed with the standard bicaval technique. In the Fontan case, a polytetra-fluor-ethylene (PTFE) graft for extension of the inferior vena cava (IVC) was used. After anastomosis of the left atrium and the aorta, reperfusion was started. The remaining anastomoses were performed during reperfusion in order to reduce ischemic time. All patient data were extracted retrospectively from the local hospital patient data capture system KISIM (CISTEC AG, Zurich, Switzerland), including cardiac diseases and comorbidities, medication, perfusion protocol, length of intensive care unit and hospital stay, ventilation times, laboratory values, pathology reports, images and microbiology. Mean follow-up was 303.25 days. Follow-up was performed in the heart transplant outpatient clinic of our center in intervals of $2-3$ weeks during the first 6 posttransplant months and every 4-6 weeks in the second half of the first posttransplant year. 

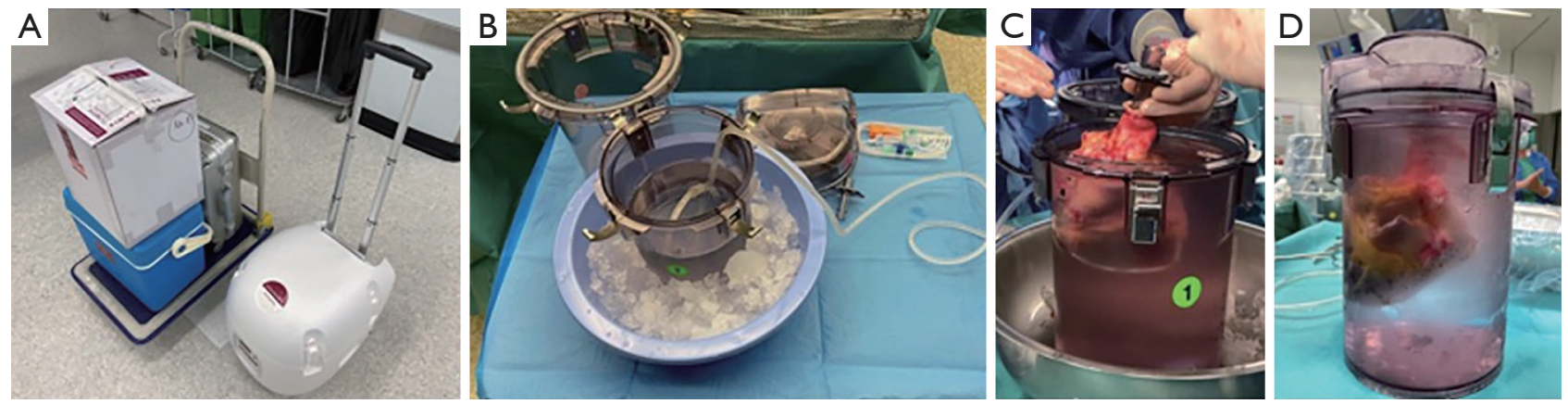

Figure 1 Images of the preparation of the SherpaPak ${ }^{\mathrm{TM}}$ Cardiac Transport System. (A) Full equipment that has to be transported to the site of organ removal; (B) during the filling procedure, the sterile inner canister is put in a bath with crushed ice to reduce the ambient temperature; (C) the aortic arch is fixed to the heart connector, allowing for free floating of the heart in the cardioplegic solution; (D) the heart is completely surrounded by cardioprotective fluid, providing mechanical protection and equal temperature to all parts of the heart. This image is published with the patient's consent.

\section{Statistical analysis}

Statistical analysis was performed using Microsoft ${ }^{\circledR}$ Excel for Mac (Microsoft, Redmond, WA, USA). Continuous variables are shown as mean \pm standard deviation and categorical data as frequencies with relative percentage $[\mathrm{n}(\%)]$.

\section{Results}

Between May and December 2020, the SherpaPak ${ }^{\mathrm{TM}}$ CTS was used in four heart transplantations. Patients' baseline characteristics are shown in Table 1. Mean age at transplantation was 50 years (range, 29-65 years). One patient was female. Two patients underwent previous cardiac surgery. From these, one patient with ischemic cardiomyopathy was supported 55 months with a centrifugal-flow LVAD type HeartWare HVAD (Medtronic, Minneapolis, MN, USA) before transplantation. The other patient suffered from a complex grown-up congenital heart disease with a failing Fontan circulation, complicated by a severe protein losing enteropathy and chronic intestinal blood losses.

Table 2 depicts the donor information. The left and right heart performance in the donor was normal in three patients and slightly reduced in patient 4 . All donors were hemodynamic stable under a low dose of noradrenalin. There was no unfavourable donor-to-recipient mismatch.

Table 3 depicts the immediate perioperative course. Mean transport distance between procurement and transplant centre was $86 \mathrm{~km}$ (range, 45-276 km). Two organ transports were carried out by helicopter and two by ambulance.
Mean storage time in the cooler was $73.5 \pm 19.33$ minutes. Total donor heart ischemic time was $199.25 \pm 11.67$ minutes. There were no incidents during transportation.

During transportation of donor hearts, probe temperature (temperature inside the innermost organ canister) as well as ambient temperature (temperature outside the shipper box) were measured continuously. The results are shown in Figure 2. Mean probe temperature during transport dropped from 8.8 to $5.2^{\circ} \mathrm{C}$, while mean ambient temperature was steady around $17^{\circ} \mathrm{C}$ (Figure $2 E$ ). The donor heart for patient 1 (Figure $2 A$ ) experienced a temperature of $14.1{ }^{\circ} \mathrm{C}$ at the beginning of transportation. Probe temperature decreased continuously during transport reaching $8.9^{\circ} \mathrm{C}$. Ambient temperature stayed stable at around $19{ }^{\circ} \mathrm{C}$. Probe temperatures of donor hearts for patients 2-4 could be held in a narrow range from $6.6^{\circ} \mathrm{C}$ to $5.2^{\circ} \mathrm{C}$ in patient 2 (Figure $2 \mathrm{~B}$ ), 8.4 to $7.6^{\circ} \mathrm{C}$ in patient 3 (Figure $2 C$ ) and 7.4 to $5.8{ }^{\circ} \mathrm{C}$ in patient 4 (Figure $2 D$ ). The respective ambient temperatures showed fluctuations in a scope of 16 to $17.7^{\circ} \mathrm{C}$ in patient $2,16.7$ to $18.7^{\circ} \mathrm{C}$ in patient 3 and 14.7 to $17.2{ }^{\circ} \mathrm{C}$ in patient 4 , reflecting the different climate conditions in the operating room and during transport in the ambulance or helicopter.

Cardiopulmonary bypass time was longer in patient 3 and 4 as compared to the other patients. Immediate postimplant left ventricular function was normal in all cases, right ventricular function was mildly reduced in all hearts. Inotropic requirement at the end of the surgery was low in patients 1 and 2 and low to moderate in patients 3 and 4 (Table 3). Tables 4,5 summarize postoperative course and adverse events. All patients completed the follow-up. 
Table 1 Patient characteristics

\begin{tabular}{|c|c|c|c|c|}
\hline Characteristics & Patient 1 & Patient 2 & Patient 3 & Patient 4 \\
\hline Age at transplantation, years & 57 & 49 & 29 & 65 \\
\hline Weight (kg) & 70.4 & 51.65 & 76.2 & 65.8 \\
\hline Diagnosis & $\begin{array}{l}\text { Dilative } \\
\text { cardiomyopathy }\end{array}$ & $\begin{array}{l}\text { Hypertrophic } \\
\text { cardiomyopathy }\end{array}$ & $\begin{array}{l}\text { Double inlet left ventricle with } \\
\text { transposition of the great vessels }\end{array}$ & $\begin{array}{l}\text { Ischemic } \\
\text { cardiomyopathy }\end{array}$ \\
\hline Previous cardiac surgery & 0 & 0 & 1 & 1 \\
\hline Previous ICD, CRT or CRT-D & 1 & 1 & 1 & 0 \\
\hline Diabetes mellitus & 1 & 0 & 0 & 1 \\
\hline Vascular disease (peripheral, carotid) & 0 & 0 & 0 & 1 \\
\hline
\end{tabular}

1, yes; 0, no. ECMO, extracorporeal membrane oxygenation; VAD, ventricular assist device; ICD, implantable cardioverter-defibrillator; CRT, cardiac resynchronization therapy; CRT-D, cardiac resynchronization therapy defibrillator; GFR, glomerular filtration rate.

Table 2 Donor information

\begin{tabular}{|c|c|c|c|c|}
\hline Characteristics & Donor 1 & Donor 2 & Donor 3 & Donor 4 \\
\hline Age, years & 50 & 33 & 17 & 52 \\
\hline Weight (kg) & 65 & 70 & 75 & 80 \\
\hline LVEF (\%) & 65 & 63 & 60 & 47 \\
\hline RV FAC (\%) & 42 & 53 & 35 & $-^{\dagger}$ \\
\hline D/R weight mismatch (\%) & -7.67 & 26.2 & -1.57 & 17.75 \\
\hline
\end{tabular}

${ }^{\dagger}$, right ventricle function was not assessed quantitatively, but was found to be slightly restricted. LVEF, left ventricular ejection fraction; RV

FAC, right ventricular fractional area change; NA, noradrenaline; D/R, donor/recipient.

Table 3 Immediate perioperative course

\begin{tabular}{|c|c|c|c|c|c|}
\hline Variables & Patient 1 & Patient 2 & Patient 3 & Patient 4 & Mean \pm SD \\
\hline Transportation time (min) & 62 & 97 & 54 & 81 & $73.50 \pm 19.33$ \\
\hline Donor heart ischemia time (min) & 189 & 201 & 215 & 192 & $199.25 \pm 11.67$ \\
\hline Recipient cross clamp time (min) & 87 & 66 & 117 & 60 & $82.5 \pm 25.75$ \\
\hline Recipient bypass time (min) & 165 & 150 & 300 & 197 & $203 \pm 67.57$ \\
\hline Immediate post-implant RV FAC (\%) & $-^{\dagger}$ & 26 & $-^{\dagger}$ & $-^{\dagger}$ & - \\
\hline
\end{tabular}

${ }^{\dagger}$, RV FAC not measured, RV function was described as mildly reduced. SD, standard deviation; Adr, adrenaline; Mil, milrinone; LVEF, left ventricular ejection fraction; RV FAC, right ventricular fractional area change. 
A

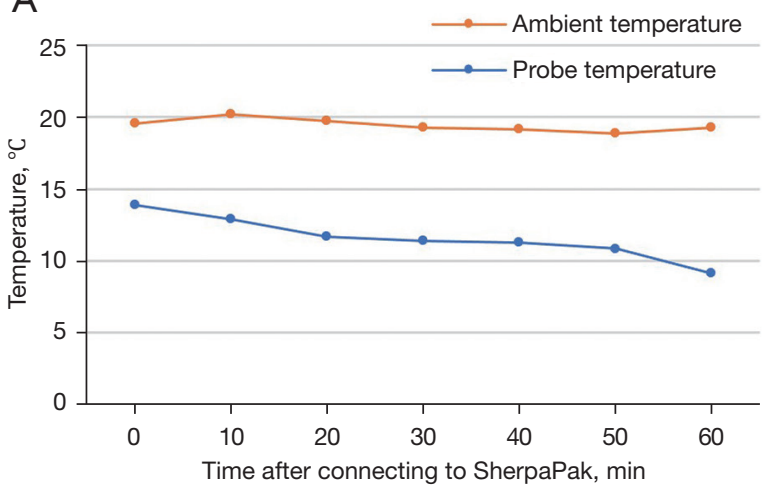

C

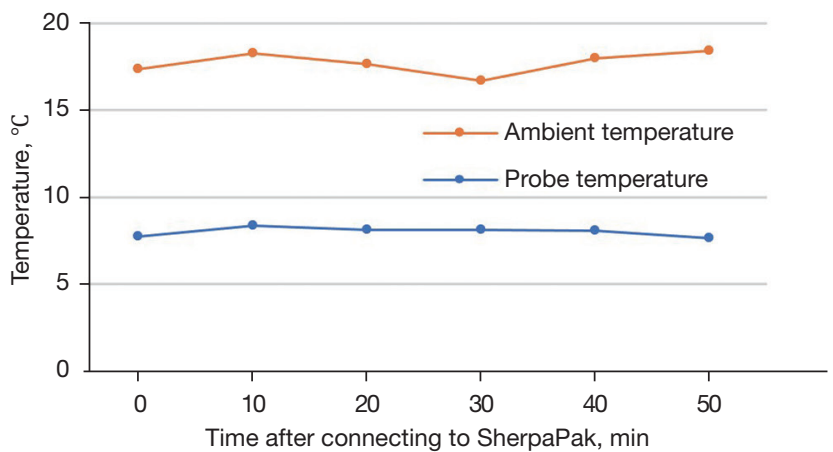

B

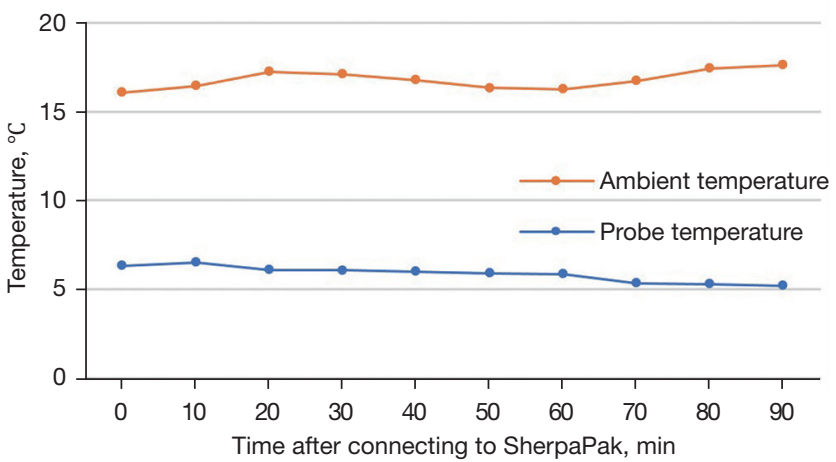

D

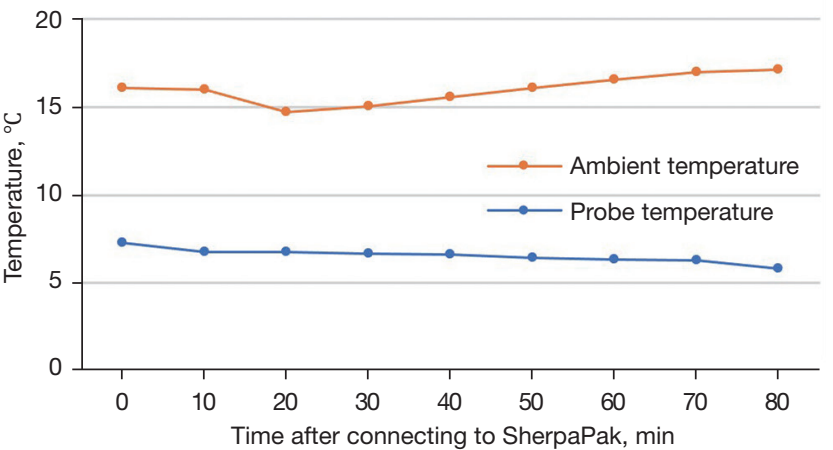

$\mathrm{E}$

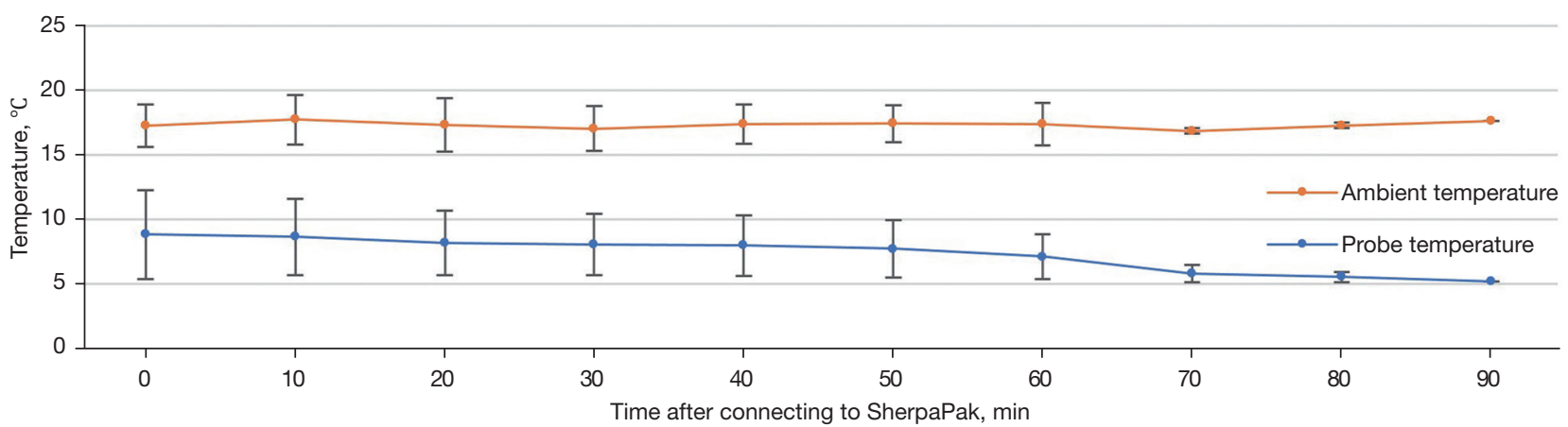

Figure 2 Temperature courses inside the organ canister (= probe temperature) and outside the shipper box (= ambient temperature) during transportation of donor hearts for patients 1-4 (A-D). (E) It depicts the mean temperature \pm standard deviation of the probe and ambient temperature of all four cases.

Length of intensive care unit (ICU) and hospital stay of patient 3 was longer than normally expected. Two patients required a temporary renal replacement therapy. Patient 1 showed a deterioration of renal function with hyperkalemia on postoperative day (POD) 1. A continuous venous-venous hemodialysis (cvvHD) was set up for three days. Patient 3 was in need of continuous venous-venous hemodiafiltration, $\mathrm{cvvHD}$ as well as intermittent hemodialysis for altogether
34 days due to acute renal insufficiency on the first POD. GI-bleeding occurred in one patient. Patient 3 presented on POD 1 with IVC kinking and stenosis due to the anatomical conditions after Fontan procedure. After rethoracotomy, a $19 \mathrm{~mm}$ PTFE tube was implanted to extend the IVC and eliminate the kinking.

Figure 3 depicts serial echocardiographic assessments throughout the follow-up. Left ventricular ejection fraction 
Table 4 Postoperative course

\begin{tabular}{|c|c|c|c|c|c|}
\hline Variables & Patient 1 & Patient 2 & Patient 3 & Patient 4 & Mean \pm SD \\
\hline Length of ICU stay (d) & 8 & $5\left(4+1^{\S}\right)$ & 21 & 7 & $10.25 \pm 7.27$ \\
\hline Length of hospital stay (d) & 31 & 51 & 111 & 35 & $57.00 \pm 37.02$ \\
\hline Alive at last follow-up (d) & 389 & 314 & 290 & 220 & $303.25 \pm 69.7$ \\
\hline
\end{tabular}

${ }^{\ddagger}$, the patient was intubated for seven days, but then reintubated for one day due to respiratory insufficiency; ${ }^{\S}$, the patient stayed on the ICU for four days, but was moved to the ICU again for 1 day due to a hemorrhagic shock. ICU, intensive care unit.

Table 5 Postoperative adverse events

\begin{tabular}{|c|c|c|c|c|c|}
\hline Adverse event & Patient 1 & Patient 2 & Patient 3 & Patient 4 & Number (\%) \\
\hline Gl-bleeding & 0 & 1 & 0 & 0 & $1(25)$ \\
\hline Renal replacement therapy [days] & $1[3]$ & 0 & $1[34]$ & 0 & $2(50)$ \\
\hline Pneumonia & 0 & 0 & 0 & 0 & $0(0)$ \\
\hline Stroke & 0 & 0 & 0 & 0 & $0(0)$ \\
\hline
\end{tabular}

", revision of inferior vena cava anastomosis. 1 , yes; 0 , no; Gl, gastrointestinal.

(LVEF) stayed within normal limits until the end of followup, except in patient 4 who experienced a drop of LVEF seven months after transplantation (Figure 3A). Right ventricular (RV) function, evaluated by fractional area change (FAC) and tricuspid annular plane systolic excursion (TAPSE), improved over time in all patients (Figure $3 B, 3 C)$. Tricuspid insufficiency at the end of follow-up was absent in one patient, mild in two patients and moderate in one patient (Figure 3D). Endomyocardial biopsies were evaluated periodically after heart transplantation using the grading system established by the ISHLT in 1990 (4). Three patients showed one moderate [ISHLT (1990) 3A] cellular rejection, treated by prednisolon pulse therapy. Otherwise, biopsies revealed no [ISHLT (1990) 0] or mild [ISHLT (1990) 1 and 2] cellular rejection. The course of the biopsy results is depicted in Figure 4.

All patients survived to hospital discharge and are currently alive (mean follow-up at the time of submission of the article: 303.25 days).

\section{Discussion}

Organ preservation is a fundamental component of heart transplantation and ensures the sustainment of organ viability. A cornerstone is cold ischemia, which begins with infusion of ice-cold cardioplegic solution after aortic cross-clamping in the donor and concludes with the start of reperfusion in the recipient. Despite its protective effect, cold ischemic storage can lead to graft injury due to activation of harmful processes resulting from ischemiareperfusion injury, including cellular swelling, intracellular acidosis, extracellular edema, calcium overload and endothelial injury (5). The current standard for donor heart preservation consists of cold organ storage in three sequential plastic bags placed in an ice box (1). The first bag contains the heart itself immersed airless in 1 litre of ice-cold preservation solution. After sealing the bag with a mersilene strap, it is put in a second bag filled with 2 litres of ice-cold isotone saline. These two bags are put in a third bag, also filled with ice-cold saline. This package is stored in a box filled with crushed ice. The saline solution in the bags is thought to prevent contact with ice and therefore minimize the risk of freezing the organ. The theoretical ideal heart temperature during transport is around $5-10{ }^{\circ} \mathrm{C}(1)$. Disadvantage of the above-described method is that the organ is cooled unevenly with parts being closer to the ice and experiencing lower temperatures eventually freezing, which may cause irreversible cellular damage 

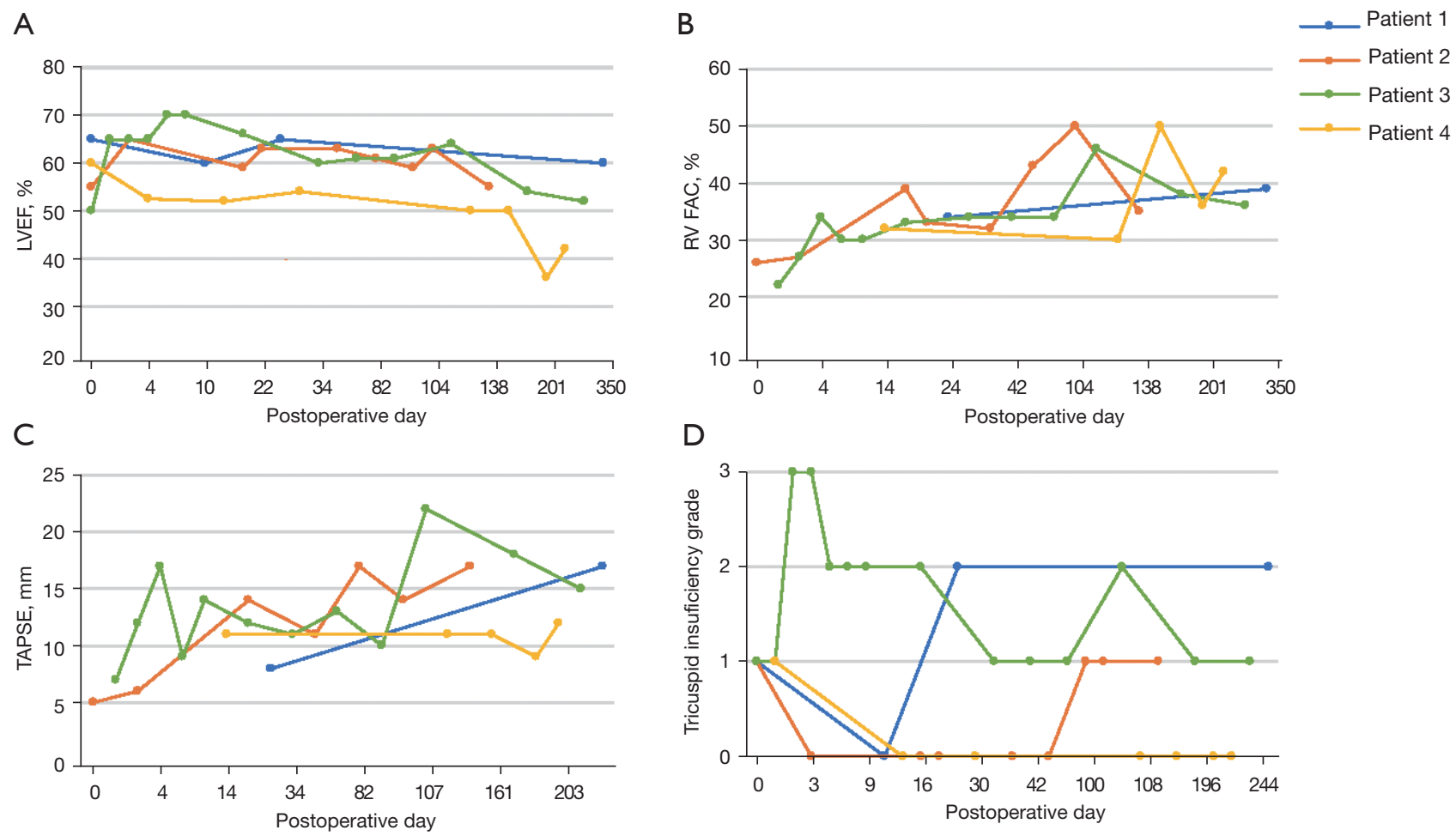

Figure 3 Serial results of echocardiographic assessment of left and right ventricular function until end of follow-up. The following parameters were evaluated: LVEF (A), RV FAC (B), TAPSE (C) and the tricuspid insufficiency grade (D) from 0 to $3(0=$ trivial, $1=$ mild, $2=$ moderate, 3 = severe). LVEF, left ventricular ejection fraction; RV FAC, right ventricular fractional area change; TAPSE, tricuspid annular plane systolic excursion.

$(1,6)$. In addition, the temperature is not monitored. Thus, low temperatures below $5{ }^{\circ} \mathrm{C}$ cannot be detected. In a multicentre study using a temperature sensor in 186 organ procurements following currently recommended packaging guidelines, the average organ temperature was below $2{ }^{\circ} \mathrm{C}$ (3). This increases the risk of hypothermic injury with protein denaturation and thereby may affect organ function after transplantation. Conversely, the increased metabolic demand at temperatures above $12{ }^{\circ} \mathrm{C}$ can lead to irreversible warm hypoxic injury of the organ.

The SherpaPak ${ }^{\mathrm{TM}}$ CTS with its CoolSafe ${ }^{\mathrm{TM}}$ technology aims to resolve these problems by maintaining a controlled preservation temperature between 4 and $8{ }^{\circ} \mathrm{C}$. Previous studies reporting clinical usage of this system have shown that throughout transportation, organ temperature can be maintained within an optimal narrow range (7-9). Even under extreme temperature conditions ( -8 and $\left.31^{\circ} \mathrm{C}\right)$, temperature in the inner container can be kept in the desired range of $4-8{ }^{\circ} \mathrm{C}(6)$. In the inner container, the heart is completely surrounded by evenly cooled cardioprotective fluid which provides an equal temperature to all parts of the heart. In addition, the two containers and the shipper box ensure mechanical protection to the organ during transport.

Between May and December 2020, we performed four heart transplantations using SherpaPak ${ }^{\mathrm{TM}}$ CTS. At first application, the initial probe temperature after logging was unexpectedly high at $14.1{ }^{\circ} \mathrm{C}$, even though appropriately cooled cardioplegic solution was used as transport medium. During transport to our centre, temperature dropped to $8.9^{\circ} \mathrm{C}$. Two main causes for the initial high temperature could be identified. First, the inner canister was filled with cooled cardioplegic solution before the heart was explanted, to ensure that the transport system is ready for housing the organ immediately after explantation. This caused a warming of the cardioplegic solution during the time of the procurement procedure. Second, the infusion system that was used to fill up the inner canister was small in diameter. Accordingly, the filling process took longer, such that the temperature of the preservation solution increased to 
A

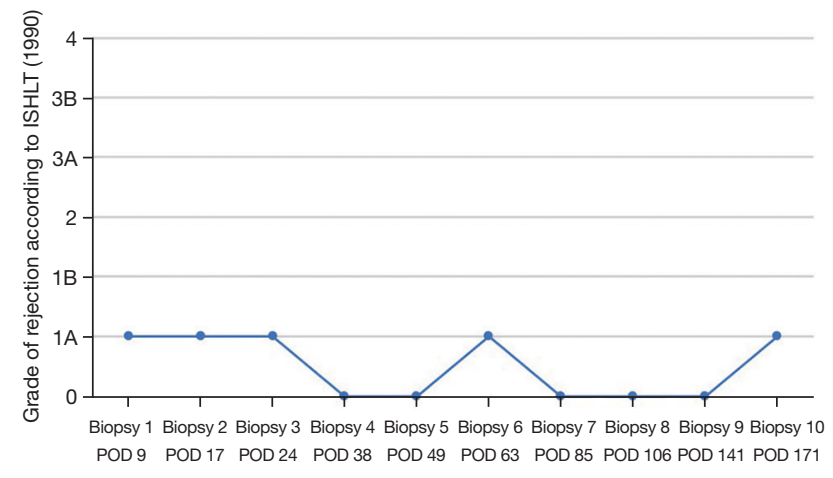

C

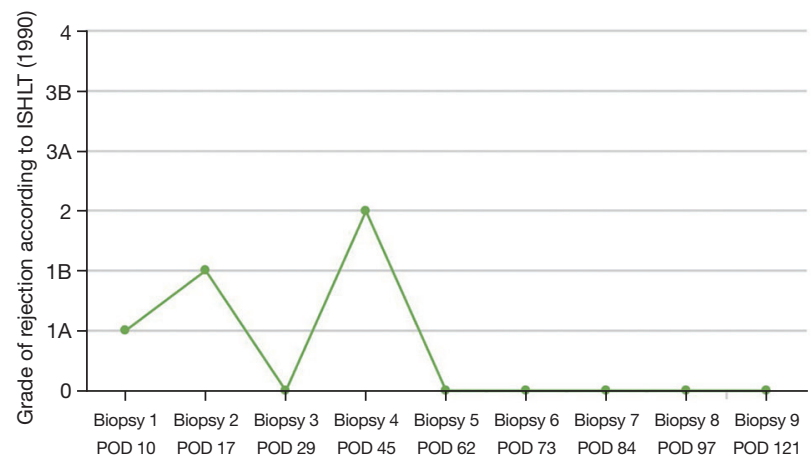

B

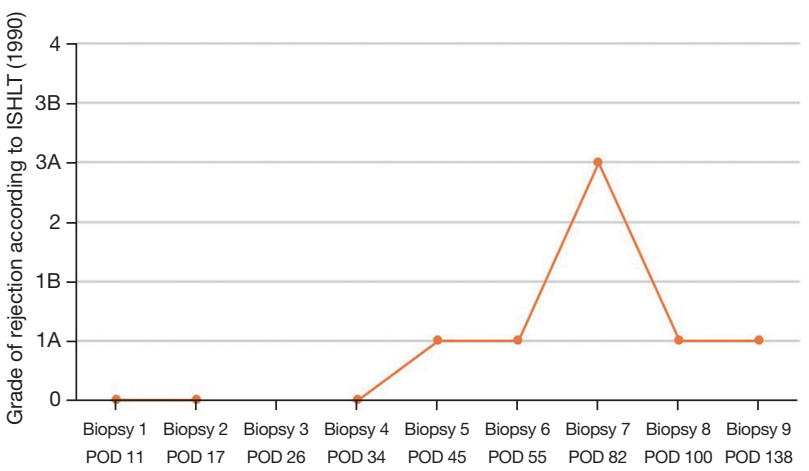

D

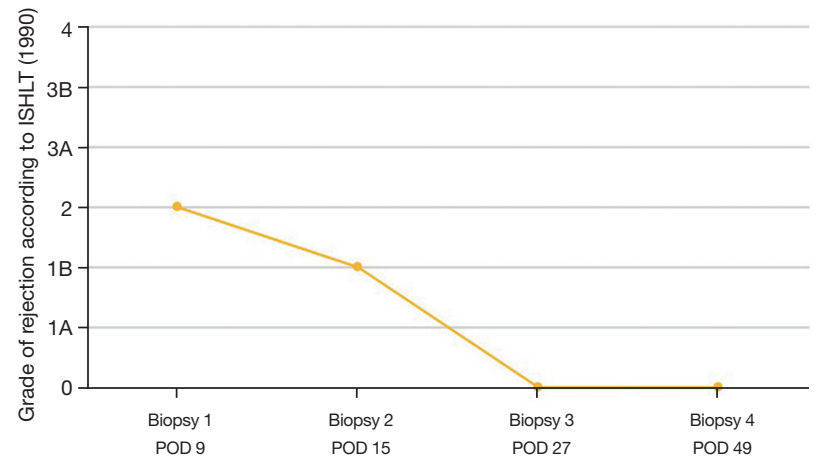

Figure 4 Course of the endomyocardial biopsy results of patient 1-4 (A-D). Comment: the third biopsy of patient 2 (B) on POD 26 did not reveal sufficient material for histological assessment according to ISHLT criteria. In the available tissue, no lymphocytic infiltrates were found. POD, postoperative day; ISHLT, International Society of Heart and Lung transplantation.

operating room temperature. We eliminated these factors by using a tubing system with a larger diameter, to speed up the filling of the canister, and by starting the filling procedure only after the heart was explanted. Both measures prevented inappropriate warming of the preservation solution. To further reduce the ambient temperature, we put the sterile inner canister in a bath with crushed ice while it was being filled (Figure 1B). In consequence, the starting probe temperature of the following procurements was in the desired range of 4 to $8{ }^{\circ} \mathrm{C}$ and stayed steady during the entire transportation time. It is fundamental that during the filling procedure, optimal target temperatures are reached in the inner canister, as the shipper does not have an active cooling mechanism. Nevertheless, the temperature dropped continuously in the first case until the end of transport, most likely caused by the passive cooling elements in the shipper box. If the initial temperature was within the target range, the system was able to maintain this temperature over the entire transport period, as demonstrated by the following three cases.
Even though the donor heart for patient 1 experienced a temperature above the upper level according to the guidelines, immediate post-implant left ventricular function was excellent. Right ventricular performance was mildly reduced, as we see frequently after transplantation. Similarly, the initial behavior of the other three hearts, showing normal left and only mildly reduced right ventricular function after reperfusion, indicated adequate preservation during ischemia. This is also underlined by the only low to moderate inotropic requirements. In the first three patients, left ventricular function remained preserved and right ventricular function improved throughout the follow-up period. Patient 4 experienced a decrease of LVEF after 7 months. In the absence of cellular and humoral rejection as well as relevant allograft vasculopathy, the etiology remains unclear so far. Diagnostics are still in progress. Remarkably enough, the left ventricular function in the donor before explantation was slightly reduced at an LVEF of $47 \%$.

The prolonged cardiopulmonary bypass time in patient 
3 and 4 can be explained by the previous cardiac operations. Accordingly, more time was required to prepare the situs for proper explantation of the recipient heart and bleeding control after transplantation, particularly in patient 3 who had undergone three former operations. The need for reoperation in patient 3 and the complexity of this patient's disease contributed to the prolonged length of ICU and hospital stay and the extended need for renal replacement therapy.

A useful tool is the InTempConnect ${ }^{\circledR}$ application for mobile phones allowing for real-time temperature measurement and tracking of the current location. This application enables the transplant surgeon to query the inside and outside temperature of the transport system at any time without having direct access to the container. The team at the transplant centre can always track the location of the organ and estimate the arrival time, helping to better synchronize the operation and thus keeping ischemic times short. With regard to temperature monitoring, it is of enormous importance that the connector of the temperature probe between the innermost and outer canister, firstly, is dry and, secondly, snapped correctly into the outer canister. Also, when fitting the outer canister into the white styrofoam transport box, it is crucial that the temperature connector is dry and properly clicked into its holder to ensure appropriate temperature monitoring.

The SherpaPak ${ }^{\mathrm{TM}}$ CTS provides progress in the field of heart preservation. However, it still is based on cold ischemic storage with all its drawbacks. There have been approaches in the past to abandon cold ischemic storage, especially with perfusion methods. Continuous hypothermic perfusion involves the infusion of a cold conservation fluid into the vasculature of the heart (10). Reports have shown that this method is superior to cold ischemic storage $(11,12)$. Still, the use of this method is limited due to logistic difficulties and high cost. In recent years, there has been increasing interest in normothermic perfusion methods. Such a method would shorten cold ischemic time and thus lower the ischemia-reperfusion injury of the graft. A report evaluating such a system showed that it is at least non-inferior to the current standard technique (13). Nevertheless, it is unlikely that it is being used in routine transplantation since it is expensive and more complicated to manage. Evidently, SherpaPak ${ }^{\mathrm{TM}}$ CTS also is more expensive than the standard three-bag-technique. In addition, the equipment that has to be transported to the site of organ procurement is bulkier (Figure 1A), which particularly is noticed when traveling by helicopter. However, these drawbacks are outweighed by the advantages of the system, such as even cooling of the organ, prevention of freezing injuries and mechanical protection.

One limitation of the study is the small study size, making it unable to generalize the results. Larger prospective trials comparing the traditional three-bag-technique with the SherpaPak ${ }^{\mathrm{TM}}$ CTS are missing. The only case-control study to date indicated that transplants preserved with SherpaPak ${ }^{\mathrm{TM}}$ CTS showed normal perioperative function (7). It even found better right ventricular function in patients in the SherpaPak ${ }^{\mathrm{TM}}$ group, suggesting that this might be the result of less hypothermic injury. Still, future research is needed to address this topic in more detail.

\section{Conclusions}

The SherpaPak ${ }^{\mathrm{TM}}$ CTS provides constant organ temperatures during transportation, prevents freezing injury and ensures mechanical protection of the graft. It allows for continuous, real-time temperature measurement and tracking of the current location. A quick filling of the inner organ canister with a correctly tempered preservation solution, starting immediately after organ procurement, is crucial to obtain the desired target temperature and to maintain it throughout transportation. Organs transported with this novel storage system showed normal postoperative function.

\section{Acknowledgments}

Funding: None.

\section{Footnote}

Reporting Checklist: The authors have completed the STROBE reporting checklist. Available at https://dx.doi. org/10.21037/jtd-21-1175

Data Sharing Statement: Available at https://dx.doi. org/10.21037/jtd-21-1175

Conflicts of Interest: All authors have completed the ICMJE uniform disclosure form (available at https://dx.doi. org/10.21037/jtd-21-1175). The authors have no conflicts of interest to declare.

Ethical Statement: The authors are accountable for all aspects of the work in ensuring that questions related 
to the accuracy or integrity of any part of the work are appropriately investigated and resolved. The study was conducted in accordance with the Declaration of Helsinki (as revised in 2013). Informed consent was obtained from all patients. An authorization from the local ethics committee (Kantonale Ethikkommission Zurich, KEK) was not required for this study since it does not fall within the scope of the Human Research Act. A written statement of the KEK is available.

Open Access Statement: This is an Open Access article distributed in accordance with the Creative Commons Attribution-NonCommercial-NoDerivs 4.0 International License (CC BY-NC-ND 4.0), which permits the noncommercial replication and distribution of the article with the strict proviso that no changes or edits are made and the original work is properly cited (including links to both the formal publication through the relevant DOI and the license). See: https://creativecommons.org/licenses/by-nc-nd/4.0/.

\section{References}

1. Copeland H, Hayanga JWA, Neyrinck A, et al. Donor heart and lung procurement: A consensus statement. J Heart Lung Transplant 2020;39:501-17.

2. Hendry PJ, Walley VM, Koshal A, et al. Are temperatures attained by donor hearts during transport too cold? J Thorac Cardiovasc Surg 1989;98:517-22.

3. Horch DF, Mehlitz T, Laurich O, et al. Organ transport temperature box: multicenter study on transport temperature of organs. Transplant Proc 2002;34:2320.

4. Billingham ME, Cary NR, Hammond ME, et al. A working formulation for the standardization of nomenclature in the diagnosis of heart and lung rejection: Heart Rejection Study Group. The International Society for Heart Transplantation. J Heart Transplant

Cite this article as: Schmiady MO, Graf T, Ouda A, Aser R, Flammer AJ, Vogt PR, Wilhelm MJ. An innovative cold storage system for donor heart transportation-lessons learned from the first experience in Switzerland. J Thorac Dis 2021;13(12):67906799. doi: $10.21037 /$ jtd-21-1175
1990;9:587-93.

5. Jahania MS, Sanchez JA, Narayan P, et al. Heart preservation for transplantation: principles and strategies. Ann Thorac Surg 1999;68:1983-7.

6. Michel SG, LaMuraglia Ii GM, Madariaga ML, et al. Innovative cold storage of donor organs using the Paragonix Sherpa Pak ${ }^{\mathrm{TM}}$ devices. Heart Lung Vessel 2015;7:246-55.

7. Radakovic D, Karimli S, Penov K, et al. First clinical experience with the novel cold storage SherpaPak ${ }^{\mathrm{TM}}$ system for donor heart transportation. J Thorac Dis 2020;12:7227-35.

8. Naito N, Funamoto M, Pierson RN, et al. First clinical use of a novel hypothermic storage system for a longdistance donor heart procurement. J Thorac Cardiovasc Surg 2020;159:e121-3.

9. Goekler J, Zuckermann A, Angleitner P, et al. First Experience with a New Storage Device for Cold Preservation. J Heart Lung Transplant 2020;39:S244.

10. Wicomb W, Cooper DK, Hassoulas J, et al. Orthotopic transplantation of the baboon heart after 20 to 24 hours' preservation by continuous hypothermic perfusion with an oxygenated hyperosmolar solution. J Thorac Cardiovasc Surg 1982;83:133-40.

11. Nutt MP, Fields BL, Belzer FO, et al. Comparison of continuous perfusion and simple cold storage for rabbit heart preservation. Transplant Proc 1991;23:2445-6.

12. Qayumi AK, Jamieson WR, Rosado LJ, et al. Preservation techniques for heart transplantation: comparison of hypothermic storage and hypothermic perfusion. J Heart Lung Transplant 1991;10:518-26.

13. Ardehali A, Esmailian F, Deng M, et al. Ex-vivo perfusion of donor hearts for human heart transplantation (PROCEED II): a prospective, open-label, multicentre, randomised noninferiority trial. Lancet 2015;385:2577-84. 